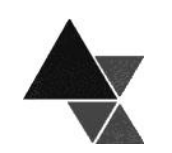

\title{
Estado nutricional de adolescentes do sudoeste do Paraná, Brasil.
}

\author{
Francini Xavier Rossetti ${ }^{1}$, Marcia Cristina Stolarski ${ }^{2}$ e Suely Teresinha Schmidt ${ }^{3}$
}

A adolescência é um período importante do desenvolvimento humano, uma vez que várias mudanças somáticas, psicológicas e sociais ocorrem visando estabelecer características do adulto. Existe consenso científico sobre a probabilidade de adolescentes obesos tornarem-se adultos obesos, com risco maior de associação a outras comorbidades, como hipertensão, dislipidemias e Diabetes Mellitus tipo 2. O objetivo deste trabalho foi avaliar o estado nutricional de adolescentes matriculados em escolas públicas estaduais em nove municípios do sudoeste do estado do Paraná (Brasil). A pesquisa foi do tipo transversal, envolvendo uma amostra de conveniência composta por 1.198 adolescentes com idade entre 10 e 19 anos. Os indicadores estabelecidos para avaliação do estado nutricional foram altura para idade e índice de massa corporal. Comparação de prevalência de excesso de peso (sobrepeso e obesidade) por faixa etária e sexo foi realizada por meio do teste qui-quadrado, assumindo $p<0,05$ como nível de significância. Os testes de correlação de Pearson e de Spearman foram realizados para examinar a existência de associação entre indicadores socioeconômicos municipais (Índice de Desenvolvimento Humano Municipal, IDH-M, Índice de Pobreza Humana Municipal, IPH-M e Índice de Gini) e o estado nutricional dos adolescentes. A prevalência de excesso de peso foi de 18,3\%; na faixa etária dos 10 aos 14 anos, $19,7 \%$ e entre os 15 e 19 anos, $13,6 \% \quad(p=0,03)$. Entre os adolescentes do sexo masculino e feminino a prevalência de excesso de peso foi: de $20,2 \%$ e $16,7 \%$, respectivamente $(p=0,15)$. Foram descritos os níveis e tendências de excesso de peso segundo os indicadores socioeconômicos municipais, porém, não foram encontrados valores significativos. A investigação de um modelo conceitual que auxilie a compreensão dos determinantes do excesso de peso em adolescentes é de fundamental importância, objetivando contribuir para a apreensão do problema em níveis tanto populacionais como regionais e definir ações e políticas públicas voltadas para o controle e prevenção nesta fase da vida.

Palavras-chave: estado nutricional, indicadores socioeconômicos, escolares, adolescentes, excesso de peso.

\section{Nutritional status of adolescents on southwestern of Paraná state (Brazil)}

Adolescence is an important period of the human development, since various changes occur - both somatic, psychological and social - aiming to establish the adult's characteristics. There is scientific consensus on the likelihood of obese adolescents become obese adults, with higher risk of association with other comorbidities, such as hypertension, dyslipidemia and diabetes melittus type 2. The aim of this research was to evaluate the nutritional status of adolescents enrolled in state public schools in 9 cities in the southwest of Paraná state (Brazil). The study was cross-sectional, involving a convenience sample of 1.198 adolescents aged between 10 and 19 years old. The indicators used for assessment of nutritional status were Height-for-age and Body Mass Index (BMI). Comparison of prevalence of excess weight (overweight and obesity) by age and sex was performed using the chi-square test, taking $p<0,05$ as significance level. Pearson and Spearman correlation tests were performed to examine the existence of association between municipal socioeconomic indicators Municipal

\footnotetext{
${ }^{1}$ Doutoranda, Pós-Graduação em Ciência e Tecnologia de Alimentos, Escola Superior de Agricultura "Luiz de Queiroz", Universidade de São Paulo. Piracicaba, SP, Brasil. Correspondência: Rua dos Sabiás no 115, Registro, SP. CEP: 11900-000. Telefone: (13) 98148-0005. E-mail: francini@usp.br

${ }^{2}$ Nutricionista, Secretaria de Estado da Educação do Paraná. Curitiba, PR, Brasil. E-mail: marcia_cs@seed.pr.gov.br

${ }^{3}$ Professora Permanente do Programa de Pós-Graduação em Segurança Alimentar e Nutricional, Universidade Federal do Paraná. E-mail: suely.ufpr@gmail.com
} 
Human Development Index (MHDI), Human Poverty Index (HPI), Gini Index and the nutritional status of adolescents. The prevalence of excess weight was $18.3 \%$; between the age groups from 10 to 14 years, $19.7 \%$ and between 15 to 18 years, $13.6 \%(p=0,030)$. Among male and female adolescents, the prevalence of weight excess were $20.2 \%$ and $16.7 \%$, respectively $(p=0,15)$. Levels and trends of excess weight were described according to the local socioeconomic indicators, however, not significant values were found. The investigation of a conceptual model to assist the comprehension the complexity of overweight determinants in adolescents is fundamentally important, aiming to contribute for the apprehension of the problem in levels both regional and population, and define actions and public policies focused on controlling and prevention at this stage of life.

Key-words: nutritional status, socioeconomic indicators, students, adolescents, weight excess.

\section{INTRODUÇÃO}

A adolescência é um período importante do desenvolvimento humano, uma vez que muitas mudanças graduais ocorrem - tanto somáticas, quanto psicológicas e sociais - visando estabelecer as características do adulto [1].

Existe consenso científico sobre a probabilidade de que a cada duas crianças obesas, uma se torne um adulto obeso [2], com risco maior de associação a outras comorbidades, como hipertensão, dislipidemia e Diabetes Mellitus tipo 2 [3,4], assim como diminuição da qualidade de vida e aumento dos gastos com saúde [5].

No Brasil, em 35 anos, a prevalência de sobrepeso e obesidade (denominadas como excesso de peso) cresceu quase seis vezes entre adolescentes do sexo masculino (de 3,7\% para $21,7 \%$ ) e mais de duas vezes entre o sexo feminino (de 7,6\% para 19,4\%) ${ }^{[6]}$.

A transição nutricional vem ocorrendo no país de modo complexo e multifacetado. Observa-se que entre adultos pertencentes aos maiores estratos de renda e especialmente entre as mulheres, a prevalência de excesso de peso vem diminuindo, ao contrário do que ocorre entre os homens em geral e os indivíduos de áreas rurais [2,7]. Entre os adolescentes das capitais brasileiras avaliados pela Pesquisa Nacional de Saúde do Escolar (PeNSE), observou-se prevalência mais elevada de excesso de peso entre aqueles com melhores condições sociais ${ }^{[8]}$.

Não obstante a transição nutricional caracterize-se pela coexistência de desnutrição e excesso de peso, o aumento da prevalência de indivíduos com sobrepeso e obesidade merece destaque, visto que realidades de vida caracterizadas por situações como fome e obesidade podem ser caracterizadas como insegurança alimentar (IA) [?].

Os métodos utilizados para monitorar e avaliar situações de IA podem ser de nível populacional, como análise de consumo de calorias per capita, pesquisas de orçamentos familiares, assim como de nível individual: antropometria, consumo de alimentos e escala de insegurança alimentar. A combinação de métodos permite capturar sob diferentes perspectivas o fenômeno da IA [10]. Além destes, as condições de vida da população podem ser mensuradas por meio de indicadores socioeconômicos como o Índice de Desenvolvimento Humano, indicador do bem-estar de uma população [11].

Buscando maior compreensão sobre perfil nutricional de adolescentes e indicadores socioeconômicos, o objetivo deste estudo foi caracterizar o estado nutricional de adolescentes matriculados em escolas públicas estaduais em nove municípios do sudoeste do Paraná, além de correlacionar a prevalência de excesso de peso e os determinantes socioeconômicos municipais, a fim de contribuir para a produção de conhecimento e discussões na área de segurança alimentar e nutricional.

\section{CASUÍSTICA E MÉTODOS}

Trata-se de uma pesquisa transversal, que integra um estudo populacional do tipo transversal, com 14.422 adolescentes e jovens beneficiários do Programa Estadual de Alimentação Escolar (PEAE), no Estado do Paraná intitulado "Estado nutricional de Escolares no Paraná", desenvolvido por meio de parceria entre a Superintendência de Desenvolvimento Educacional (SUDE), integrante da Secretaria de 
Estado da Educação do Paraná e o Departamento de Nutrição da Universidade Federal do Paraná (UFPR).

A definição da amostra foi estabelecida pela SUDE, considerando a abrangência e características do PEAE no Paraná. Em cada um dos 32 Núcleos Regionais de Educação (NRE) foram definidas as unidades de pesquisa (municípios e escolas) e o tamanho da amostra por meio de procedimento não probabilístico por conveniência. Em cada NRE, três municípios de pequeno, médio e grande porte ${ }^{4}$ foram selecionados. Todas as escolas dos municípios de pequeno porte com duas a três escolas compuseram a amostra. Para os municípios de médio e grande porte, foi selecionada a escola com menor número de estudantes, aquela com número intermediário e a escola com maior número.

Em cada escola, uma turma de 5a a 8a série do ensino fundamental e uma turma da 1a a $3 \underline{a}$ série do ensino médio foi escolhida aleatoriamente para a coleta dos dados antropométricos. Participaram do estudo 99 municípios e 324 escolas. A amostra do Sudoeste Paranaense ${ }^{5}$ foi composta por 9 municípios e 1.198 adolescentes de 10 a 19 anos.

Os dados antropométricos foram coletados em 2004, sob responsabilidade da SUDE, por nutricionistas e estudantes de nutrição previamente treinados. Os indivíduos foram pesados vestindo roupas leves e medidos sem calçados.

Os dados foram duplamente digitados, por diferentes digitadores, utilizando o programa Epi-Info versão $6 \mathrm{bc}$, e erros foram detectados e corrigidos por meio do módulo $V$ alidate.

O índice altura para idade foi utilizado para avaliar o déficit de altura e a partir das medidas de peso e altura foi calculado o Índice de Massa Corporal, por meio da fórmula $\mathrm{IMC}=$ peso $(\mathrm{kg}) /$ altura $^{2}(\mathrm{~m})$. Para a classificação dos dados, foram utilizados os valores de referência da Organização Mundial da Saúde, de acordo com o sexo e idade. Os pontos de corte do escore $Z$ para altura para idade (ZAI) foram: $Z A I<-$ 2 , altura baixa para a idade, $Z A I \geq-2$, altura adequada para a idade. Os pontos de corte do escore $Z$ para o
IMC foram: escore $Z \leq-2$, IMC abaixo do esperado para a idade, $-2<Z<1$, IMC adequado ou eutrófico, $\geq 1 \quad Z<2$, sobrepeso, $Z \geq 2$, obesidade [12]. As categorias sobrepeso e obesidade foram somadas e o desfecho denominado excesso de peso.

A variável dependente adotada para a análise foi o Índice de Massa Corporal (IMC), expresso em escore $Z$. As variáveis independentes foram IDH-M, o Índice de Gini e o IPH-M. Os dois primeiros índices foram obtidos por meio de publicações do Instituto Paranaense de Desenvolvimento Social [13,14] e o terceiro a partir de dados secundários do Atlas de Desenvolvimento Humano no Brasil [15]. O IPH-M, adaptado do Índice de Pobreza Humana foi calculado de acordo com Rolim [16] a partir do percentual de pessoas com expectativa de vida inferior a 40 anos (a), taxa de analfabetismo de adultos (b), percentual de pessoas que vivem em domicilio sem água encanada (c), e percentual de crianças até 5 anos de idade falecidas - proxy de desnutrição (d), por meio da seguinte equação:

$$
\begin{aligned}
& \text { IPH-M }=\left[1 / 3\left(a^{3}+b^{3}+e^{3}\right)\right]^{1 / 3} \\
& \text { Onde (e) }=(c)+(d) / 2
\end{aligned}
$$

Para classificação e análise dos dados antropométricos foi utilizado o programa Excel. As análises estatísticas foram realizadas com auxílio do programa Sweave. Foi realizada análise descritiva por meio do cálculo de média, mediana e desvio-padrão; para comparação dos resultados relativos ao IMC para sexo e faixa etária aplicou-se o teste do qui-quadrado.

Para avaliar a correlação entre a prevalência de excesso de peso e as variáveis socioeconômicas foram utilizados os testes paramétrico de Pearson e o teste não paramétrico de Spearman.

O estudo foi aprovado pelo Comitê de Ética do Setor de Ciências da Saúde da UFPR, sob o parecer no 232 de 2006.

${ }^{4}$ No tocante ao número de estabelecimentos de ensino público, foram classificados como de pequeno porte municípios que apresentavam de 2 a 4 escolas; médio porte aqueles que possuíam entre 5 e 10 escolas e grande porte aqueles que possuíam mais de 11 escolas.

5 Pelo fato da amostra ter sido coletada em 2004, consideraremos a microrregião de Palmas como pertencente à região Sudoeste (esta foi desanexada da mesorregião em 2005). 


\section{RESULTADOS}

A amostra do presente estudo foi composta por 1.198 adolescentes com idade entre 10 e 19 anos, dos quais $628(52,4 \%)$ eram do sexo feminino.
A prevalência de altura baixa para idade foi de $1,34 \%$, resultado menor do que o esperado de aproximadamente $2 \%{ }^{[12]}$.

Na Tabela 1 pode-se visualizar a distribuição do estado nutricional por sexo e faixa etária.

Tabela 1. Estado nutricional dos adolescentes, segundo IMC, sexo e faixa etária. Sudoeste, PR - 2004.

\begin{tabular}{|c|c|c|c|c|c|c|}
\hline \multirow{3}{*}{ Sexo } & \multirow{3}{*}{$\begin{array}{c}\text { Estratos de } \\
\text { idade (em anos) }\end{array}$} & \multicolumn{4}{|c|}{ Estado nutricional } & \multirow{3}{*}{ Valor de $(p)^{*}$} \\
\hline & & $Z \leq-2$ & $-2 Z<1$ & $Z \geq 1$ & Observações & \\
\hline & & $\mathrm{n}(\%)$ & $\mathrm{n}(\%)$ & $\mathrm{n}(\%)$ & $\mathrm{n}$ & \\
\hline \multirow{3}{*}{ Feminino } & $10-14$ & $24(4,6)$ & $403(77,8)$ & $91(17,6)$ & 518 & \multirow{3}{*}{0,12} \\
\hline & 19 & $2(1,8)$ & $94(85,5)$ & $14(12,7)$ & 110 & \\
\hline & Total & $26(4,1)$ & $497(79,1)$ & $105(16,7)$ & 628 & \\
\hline \multirow{3}{*}{ Masculino } & 14 & $11(2,6)$ & $319(75,2)$ & $94(22,2)$ & 424 & \multirow{3}{*}{0,32} \\
\hline & $14-19$ & $2(1,4)$ & $123(84,2)$ & $21(14,4)$ & 146 & \\
\hline & Total & $13(2,3)$ & $442(77,5)$ & $115(20,2)$ & 570 & \\
\hline \multirow{3}{*}{ Total } & 19 & $34(3,6)$ & $723(76,8)$ & $185(19,7)$ & 942 & \multirow{3}{*}{0,036} \\
\hline & $14-19$ & $4(1,6)$ & $217(84,8)$ & $35(13,6)$ & 256 & \\
\hline & Total & $38(3,2)$ & $940(78,5)$ & $220(18,3)$ & 1.198 & \\
\hline
\end{tabular}

Observa-se diferença estatística $(p<0,05)$ entre a prevalência de excesso de peso e os estratos de idade para a totalidade da população.
A Tabela 2 aponta o estado nutricional e altura média com respectivos desvios-padrões e medianas e a tabela 3 indica os valores relativos à altura média e a mediana de adolescentes com excesso de peso.

Tabela 2. Estado nutricional, altura média e desvio padrão, de adolescentes de 10 a 19 anos, Sudoeste, PR - 2004.

\begin{tabular}{c|c|c|c|c|c|c|c}
\hline Sexo & $\begin{array}{c}\text { Estratos de } \\
\text { idade (em anos) }\end{array}$ & $\begin{array}{c}\text { IMC } \\
\text { médio }\end{array}$ & $\begin{array}{c}\text { Desvio } \\
\text { padrão }\end{array}$ & $\begin{array}{c}\text { IMC } \\
\text { mediano }\end{array}$ & $\begin{array}{c}\text { Altura média } \\
(\mathrm{cm}) \pm \text { DP }\end{array}$ & $\begin{array}{c}\text { Desvio } \\
\text { padrão }\end{array}$ & $\begin{array}{c}\text { Altura } \\
\text { mediana }\end{array}$ \\
\hline Feminino & $10 \dashv 14$ & 18,87 & 3,12 & 18,26 & 156,6 & 8,16 & 157,0 \\
& $14-19$ & 21,25 & 2,86 & 20,88 & 162,5 & 6,12 & 162,5 \\
Masculino & $10 \dashv 14$ & 18,61 & 3,08 & 18,00 & 157,6 & 10,28 & 157,5 \\
& $14-19$ & 20,82 & 2,62 & 20,34 & 172,4 & 6,84 & 172,0 \\
\hline
\end{tabular}


Tabela 3. Altura média e mediana de adolescentes de 10 a 19 anos com sobrepeso e obesidade. Sudoeste, PR - 2004.

\begin{tabular}{c|c|c|c|c}
\hline Sexo & $\begin{array}{c}\text { Estratos de idade } \\
(\mathrm{em} \text { anos })\end{array}$ & Altura média & Desvio padrão & $\begin{array}{c}\text { Altura } \\
\text { mediana }\end{array}$ \\
\hline Feminino & $10 \dashv 14$ & 158,70 & 6,44 & 159,0 \\
& $14-19$ & 164,42 & 4,70 & 164,5 \\
Masculino & $10-14$ & 159,92 & 7,86 & 160,0 \\
& $14-19$ & 174,19 & 8,26 & 173,0 \\
\hline
\end{tabular}

A altura dos adolescentes da totalidade da amostra (Tabela 2), comparada à daqueles que apresentaram excesso de peso (Tabela 3), indica que tanto a altura média quanto a mediana foi superior à da população de estudo, com exceção para faixa etária dos 10 a 14 anos, para o sexo feminino.
O Quadro 1 apresenta os indicadores socioeconômicos por município do sudoeste paranaense.

Quadro 1. Indicadores socioeconômicos dos municípios. Sudoeste, PR - 2004.

\begin{tabular}{|c|c|c|c|}
\hline Municípios & Índice de Gini, 2000 & IDH-M, 2000 & IPH-M, 2000 \\
\hline Clevelândia & 0,56 & 0,73 & 11,62 \\
Cruzeiro do Iguaçú & 0,55 & 0,74 & 13,55 \\
Dois Vizinhos & 0,54 & 0,77 & 9,22 \\
Enéas Marques & 0,60 & 0,78 & 8,68 \\
Mangueirinha & 0,68 & 0,75 & 12,36 \\
Nova Esperança do Sudoeste & 0,56 & 0,72 & 13,08 \\
Pato Branco & 0,57 & 0,85 & 5,90 \\
Realeza & 0,55 & 0,78 & 9,02 \\
Santa Izabel do Oeste & 0,57 & 0,77 & 13,79 \\
\hline
\end{tabular}

Fonte: Ipardes ${ }^{[13,14]}$, PNUD ${ }^{[15]}$.

Em relação ao IDH, o estado do Paraná apresentou valor de 0,787 , maior do que o encontrado no Brasil (0,766). Nesse estado, entre 1999 e 2000, o componente que mais contribuiu para o seu desempenho foi a educação e o componente com ganhos mais intensos no período foi a esperança de vida ao nascer ${ }^{[14]}$.

De modo geral, o comportamento do IDH$\mathrm{M}$ foi bastante similar entre os nove municípios, com exceção de Pato Branco, que apresentou valor de 0,85 , classificado como município com alto desenvolvimento humano. Os demais foram classificados na categoria de médio desenvolvimento humano.

No tocante ao índice de GINI, entre os nove municípios, somente em Clevelândia e Mangueirinha a desigualdade de renda medida por meio do Coeficiente de Gini aumentou entre 1991 e 2000. Nos outros municípios, a desigualdade permaneceu estável ou diminuiu [15]. 
Os resultados para a melhor e a pior colocação do indicador IPH-M entre os municípios brasileiros foram: São Caetano do Sul - SP, com um IPH-M de 2,52 e Guaribas - PI, com a pior colocação, com resultado de 56,23 [17]. Para os municípios do Sudoeste do estado do Paraná inclusos na pesquisa, o índice variou de 5,9 em Pato Branco a 13,79 em Santa Izabel do Oeste. Este indicador pode ser analisado como o percentual de pessoas que vivem em condições de pobreza ${ }^{[1]}$.

$\mathrm{Na}$ Tabela 4, observa-se os resultados referentes ao estado nutricional dos adolescentes, discriminados por municípios.

Tabela 4. Estado nutricional, segundo o IMC, de adolescentes de ambos os sexos, de 10 a 19 anos, Sudoeste, PR $-2004$.

\begin{tabular}{l|rr|rr|rrrr}
\hline \multirow{2}{*}{ Municípios } & \multicolumn{2}{c}{$Z \leq-2$} & \multicolumn{2}{c}{$-2 Z<1$} & \multicolumn{2}{c}{$Z \geq 1$} & \multicolumn{2}{c}{ Observações } \\
\cline { 2 - 9 } & $\mathrm{n}$ & $\%$ & $\mathrm{n}$ & $\%$ & $\mathrm{n}$ & $\%$ & $\mathrm{n}$ & $\%$ \\
\hline Clevelândia & 2 & 1,54 & 106 & 81,54 & 22 & 16,90 & 130 & 10,85 \\
Cruzeiro do Iguaçú & 2 & 2,86 & 51 & 72,86 & 17 & 24,30 & 70 & 5,84 \\
Dois Vizinhos & 2 & 1,32 & 118 & 78,15 & 31 & 20,50 & 151 & 12,60 \\
Enéas Marques & 4 & 2,84 & 105 & 74,47 & 32 & 22,70 & 141 & 11,76 \\
Mangueirinha & 4 & 3,54 & 90 & 79,65 & 19 & 16,90 & 113 & 9,43 \\
Nova Esperança do Sudoeste & 6 & 4,48 & 105 & 78,36 & 23 & 17,20 & 134 & 11,18 \\
Pato Branco & 9 & 5,29 & 122 & 71,76 & 39 & 22,90 & 170 & 14,19 \\
Realeza & 7 & 4,20 & 131 & 78,90 & 28 & 16,86 & 166 & 13,85 \\
Santa Izabel do Oeste & 3 & 2,44 & 110 & 89,43 & 10 & 8,13 & 123 & 10,26 \\
\hline \multicolumn{1}{c}{ Total } & 39 & 3,25 & 938 & 78,30 & 221 & 18,44 & 1.198 & 100,00 \\
\hline
\end{tabular}

A prevalência de excesso de peso variou de $8,13 \%$ em Santa Izabel do Oeste a $24,3 \%$ no município de Cruzeiro do Iguaçú.

Os níveis de associação entre os indicadores socioeconômicos municipais tendo como desfecho a prevalência de excesso de peso podem ser visualizados na Tabela 5. 
Tabela 5. Coeficiente de correlação de Pearson e de Spearman e valores ( $p$ ) entre as variáveis socioeconômicas e a prevalência de excesso de peso nos municípios. Sudoeste, PR - 2004.

\begin{tabular}{c|cccc}
\hline \multirow{2}{*}{ Indicadores } & \multicolumn{4}{c}{ Prevalência de excesso de peso } \\
\cline { 2 - 4 } & \multicolumn{2}{|c}{ Correlação de Pearson } & \multicolumn{2}{c}{ Correlação de Spearman } \\
\hline \multirow{2}{*}{ IPH-M } & R & -valor & $r$ & $p$-valor \\
IDH-M & $-0,48$ & 0,19 & $-0,3$ & 0,44 \\
GINI & 0,22 & 0,57 & 0,06 & 0,88 \\
& $-0,10$ & 0,79 & $-0,14$ & 0,73 \\
\hline
\end{tabular}

A associação e tendência entre prevalência de excesso de peso e os indicadores socioeconômicos (IDH-M, IPH-M e Índice de Gini) foi verificada por meio dos testes de Spearman e Pearson (Tabela 5). De acordo com o teste de Spearman, a correlação entre o IPH-M e a prevalência de excesso de peso apontou tendência negativa $(r=-0,48)$, quanto menor o valor do índice nos municípios avaliados, maior foi a tendência de excesso de peso; porém essa associação não demonstrou ser estatisticamente significante $(p=0,19)$. $O$ índice de Gini apresentou tendência negativa $(r=-0,10)$ e não significante em relação à prevalência de sobrepeso e obesidade $(p=0,79)$ e finalmente o IDH-M apontou tendência positiva $(r=0,22)$, porém não significativa $(p=0,57)$ em relação ao percentual de adolescentes com excesso de peso.

\section{DISCUSSÃO}

A transição nutricional no Brasil caracteriza-se pela coexistência de excesso de peso e desnutrição, embora o aumento da prevalência do excesso de peso esteja crescendo [0].

A Organização Mundial da Saúde [12] recomenda que a avaliação do estado nutricional de adolescentes a nível coletivo combine os índices altura para idade e IMC uma vez que altura baixa para idade (déficit linear) pode se caracterizar como um fator de risco para o excesso de peso [18]. Todavia são poucos os estudos que utilizam os índices em conjunto [9,19].

O crescimento linear dos adolescentes do Sudoeste paranaense revelou-se em consonância às recomendações da OMS [12] demonstrando que déficit de altura não se manifesta como um problema nutricional, resultado similar ao encontrado por Bleil et al. [20] ao avaliarem escolares de um município da mesma região, encontraram prevalência de baixa altura de $1,75 \%$. Resultados referentes ao crescimento linear da totalidade da amostra de escolares paranaenses da qual derivou esta pesquisa foi avaliado no estudo de Amorim et al. [21], no qual observou-se baixa prevalência de déficit de altura, da ordem de 0,05 a 2,39 $\mathrm{cm}$ na faixa dos 12 aos 18 anos entre 13.216 escolares.

A baixa prevalência de déficit de altura encontrada nesta amostra corrobora para uma análise apropriada do estado nutricional, uma vez que prevalência de altura baixa para a idade poderia resultar em uma classificação inadequada do IMC.

Monteiro et al. [22] referem que a obesidade no Brasil não deve ser mais considerada uma doença da elite. Entre 1974 e 2008, a prevalência de excesso de peso entre adolescentes cresceu mais de seis vezes (de $3,7 \%$ para $25,4 \%$ ). No tocante às macrorregiões, o sul do país vem apresentando tendência de incremento do estado nutricional ainda maior do que apontam os dados nacionais. Em 2008-2009, 34,5\% apresentavam excesso de peso. O baixo percentual de déficit de peso encontrado nesta pesquisa (3,71\%) está de acordo com os dados encontrados pela POF de 2008-2009, que foi de $3,7 \%[7]$.

Pesquisas de base populacional com escolares adolescentes, como a Pesquisa Nacional de Saúde do Escolar (PeNSE) realizada em 2009 em todas as capitais brasileiras, registrou excesso de peso entre $30,2 \%$ dos adolescentes. Este esteve associado à macrorregião (destaque para as regiões sul e sudeste), ao sexo (masculino), ao estrato de idade (indivíduos entre 11 e 13 anos de idade são mais suscetíveis do que os mais velhos) e maior escolaridade materna ${ }^{[8]}$. Veiga, Cunha e Sichieri [7] pesquisando tendência de sobrepeso entre adolescentes no sul e nordeste do país 
concluíram que os indivíduos do sexo masculino, assim como meninas da faixa etária dos 10 aos 14 anos, tanto ricos como pobres, encontravam-se mais vulneráveis ao processo obesogênico; entretanto, entre as garotas de 15 a 19 anos da região sul, a prevalência de sobrepeso estava diminuindo, tendência que também foi observada no presente estudo.

A prevalência de excesso de peso entre os escolares do sudoeste do estado do Paraná (18,3\%) foi maior do que a encontrada entre adolescentes de Salvador - BA (14,3\%) [23] e menor do que a encontrada entre adolescentes matriculados em escolas públicas do sudeste do Brasil, como em Montes Claros - MG, (18,5\%) [24], Belo Horizonte - MG, (21,9\%) [25], Niterói - RJ, (25,7\%) [26] e Sorocaba - SP $(38,3 \%)^{[27]}$ e Guariba - SP $(38,3)^{[28] .}$

Com base na análise do estado nutricional de adolescentes por meio do IMC, pesquisadores da área vêm observando crescimento de excesso de peso de modo mais acelerado entre o sexo masculino. De acordo com o Estudo Nacional de Despesas Familiares realizado em 1974-1975, entre meninos, a prevalência de excesso de peso foi de 4,8\% e 10,6\% entre o sexo feminino. Na década seguinte, a Pesquisa de Padrões de Vida (1996-1997), indicou prevalência de sobrepeso nos garotos de $11,8 \%$ versus $15,3 \%$ entre o sexo feminino. A POF de 2008-2009 apontou prevalência de excesso de peso entre meninos de $27,3 \%$ e $23,4 \%$ entre o sexo feminino. Desde $1974-$ 1975 houve um aumento de mais de 5 vezes na prevalência de excesso de peso entre os meninos e mais de 2 vezes entre as meninas [7].

Neste estudo a prevalência de excesso de peso entre adolescentes do sexo masculino e feminino foi de $20,2 \%$ e $16,7 \%$, respectivamente. Levantamentos nacionais, como a POF de 2008-2009 e a Pesquisa Nacional de Saúde do Escolar (PeNSE) [?] também apontaram indivíduos do sexo masculino com maior prevalência de excesso de peso.

Em concordância com os resultados da presente pesquisa e dados nacionais, pesquisas de base escolar realizada por Silva et al. [29], Costa, Cordini Junior e Matsuo [30] observaram maiores prevalências de excesso de peso no sexo masculino em relação ao feminino, porém não estatisticamente significativos. Tendência observada também por Castilho et al. ${ }^{[31]}$, e ainda, Bertin et al. [32], Frainer et al. [23], Farias Junior et al. [33], Peres et al. [34], Costa et al. [35], Souza et al. [27] apontaram resultados similares, porém nestes estudos não houve análise estatística comparando sexo. Ao analisar uma coorte nacional com de adolescentes entre 11 e 14 anos de idade entre os anos de 2005 e 2011, Flores et al. [36] observou o aumento da prevalência de excesso de peso entre meninos (de 26,3\% para 29,6\%) e declínio entre meninas (de 30,7\% para $28 \%$ ).

Resultados distintos no que se refere a maior prevalência de excesso de peso entre indivíduos do sexo masculino foram observados entre as pesquisas de alcance regional realizadas com escolares por Bispo et al. [25], na qual encontraram prevalência de excesso de peso maior entre o sexo feminino $(p<0,01)$, assim como Pinho et al. ${ }^{[24]}(p=0,276)$.

A tendência do incremento do excesso de peso entre meninos, pode ser atribuída a alguns fatores, como a busca de ganho de massa muscular e consequentemente de peso, enquanto meninas objetivam corpos mais magros [34]; ou ainda, superestimação do excesso de peso, uma vez que o IMC pode refletir não somente excesso de gordura, mas músculos e outros tecidos corpóreos [37]. Outra hipótese seria que a prevalência de excesso de peso entre o sexo feminino é uma realidade nos segmentos em piores condições sociais ou regiões menos desenvolvidas [38].

Analisando os resultados por faixa etária, observa-se que maior ocorrência de excesso de peso incide entre a faixa menor de idade: 19,7\%, comparado ao grupo etário de 15 a 19 anos de idade: 13,64\% $(p<0,05)$. Resultados similares foram encontrados por Duncan et al. [39]: 25,6\% x 16,1\% e Vasconcellos et al. [26], em que a prevalência de excesso de peso entre os adolescentes de 10 a 14 anos foi quase 2 vezes maior comparada aos adolescentes mais velhos: $31,5 \% \mathrm{x}$ $16,2 \%(p<0,05)$. Pesquisa realizada por Castilho et al. [31], apontou chance $60 \%$ maior de adolescentes mais jovens (de 11 a 14 anos) apresentarem excesso de peso comparados aos indivíduos de 15 a 18 anos de idade. Guedes et al. ${ }^{[37]}$ observaram prevalência ligeiramente menor no grupo etário mais jovem $(12,1 \% \times 13,2 \%)$, assim como Frainer et al. [23] (12,9 x 15,3\%).

De acordo com Silva, Balaban e Motta [40] a tendência de excesso de peso entre os adolescentes mais jovens é esperada; porém o crescimento previsto pelo estirão pubertário ocorre a possibilidade de normalizar o estado nutricional. Duncan et al. ${ }^{[39]}$ citam que adolescentes podem ser mais expostos a ambientes não saudáveis durante a primeira infância 
quando comparado à infância tardia e adolescência. Vasconcellos et al. ${ }^{[26]}$ afirmam que o excesso de peso entre os mais jovens pode decorrer da maturação sexual ou ainda de condições socioeconômicas, merecendo aprofundamento da questão.

A identificação e análise dos aspectos econômicos, políticos e culturais faz-se imprescindível para compreender de que maneira os modos de vida da população em estudo se refletem em seu estado de nutrição. Os determinantes no nível municipal que definem as condições de vida intermeiam a relação de acesso aos bens e serviços e se expressam direta ou indiretamente nas condições de saúde e nutrição do adolescente [11].

As variáveis socioeconômicas municipais objetivam captar condições de vida da população sob diferentes enfoques [16]. Estes indicadores têm como premissa determinar as condições da estrutura social, seja ela necessidades básicas (medida através do IDHM), capacitação humana (medida através do IPH-M), políticas públicas oferecidas pela esfera governamental (medida através do índice de GINI), que se materializam nas condições socioeconômicas e manter estreita relação com o estado nutricional de uma comunidade ${ }^{[11]}$.

Oliveira et al. [11], ao analisarem os determinantes do crescimento linear em crianças utilizando a modelagem multinível (dados referentes os municípios, ao domicílio e a própria criança) encontraram que determinantes ao nível do município - IDH-M, assistência pré-natal - explicaram em 15,7\% a variabilidade à desnutrição.

\footnotetext{
A associação entre excesso de peso e os indicadores socioeconômicos municipais demonstraram tendências de correlação, mas não foram estatisticamente significantes. $\mathrm{O}$ pequeno número de municípios na amostra pôde não ser capaz de correlacionar com clareza estatística sobre as condições de vida e o estado nutricional dos adolescentes. Porém, esses resultados não devem ser generalizados para adolescentes de outras regiões da pesquisa, pelo fato da amostra não probabilística e também pelas características que podem ser encontradas entre adolescentes de diferentes regiões.
}

\section{CONCLUSÕES}

O estado nutricional de adolescentes dos municípios do Sudoeste Paranaense segue a tendência do incremento da prevalência de sobrepeso e obesidade assim como vem ocorrendo entre os adolescentes do restante do país. Entre os indivíduos do sexo masculino, o excesso de peso foi de $20,7 \%$ e entre o sexo feminino, $16,7 \%(p=0,15)$. A análise dos resultados por faixa etária demonstra que maior prevalência de excesso de peso ocorreu na faixa menor de idade: $19,7 \%$, seguido por $13,64 \%$ para o grupo etário entre 15 e 19 anos $(p=0,03)$.

Os níveis e tendências entre IDH-M, IPH-M e índice de Gini, correlacionados com a prevalência de excesso de peso não resultaram em valores estatisticamente significativos de associação. A investigação de um modelo conceitual que melhor compreenda a complexidade dos determinantes do excesso de peso em adolescentes é de fundamental importância, objetivando contribuir para a apreensão do problema em níveis tanto populacionais como regionais e definir ações e políticas públicas na área de segurança alimentar e nutricional voltadas para o controle e prevenção nesta fase da vida.

\section{AGRADECIMENTOS}

Agradecemos ao Prof. Dr. Joel da Rosa Correa (The Rockfeller University/Universidade Federal do Paraná) pelo auxílio nas análises estatísticas.

\section{REFERÊNCIAS}

[1] World Health Organization. Nutrition in adolescence issues and challenges for the health sector: issues in adolescent health and development. Geneva: World Health Organization; 2005. 115p.

[2] Conde WL, Borges C. O risco de incidência e persistência da obesidade entre adultos Brasileiros segundo seu estado nutricional ao final da adolescência. Rev. Bras. Epidemiol. 2011;14 (suppl.1):71-79.

[3] Dietz WH. Health consequences of obesity in youth: Childhood predictors of adult disease. Pediatrics.1998;101Supl:518-25. 
[4] Costa RS, Sichieri R. Relação entre sobrepeso, adiposidade e distribuição de gordura com a pressão arterial de adolescentes no município do Rio de Janeiro. Rev Bras Epidemiol. 1998;1(3):268-79.

[5] Frederick CB, Snellman K, Putnam RD. Increasing socioeconomic disparities in adolescent obesity. Proc Natl Acad Sci U S A. 2014;111(4):1338-42.

[6] Instituto Brasileiro de Geografia e Estatística. Pesquisa de Orçamentos Familiares 2008-2009: antropometria e estado nutricional de crianças, adolescentes e adultos no Brasil. Rio de Janeiro: Instituto Brasileiro de Geografia e Estatística; 2010. $121 \mathrm{p}$.

[7] Veiga GV, Cunha AS, Sichieri, R. Trends in Overweight Among Adolescents Living in the Poorest and Richest Regions of Brazil. Am J Public Health. 2004;94(9):1544-8.

[8] Araujo C, Toral N, Silva ACF, Melendez GV, Dias AJR. Estado nutricional dos adolescentes com variáveis sociodemográficas: Pesquisa Nacional de Saúde do Escolar (PeNSE), 2009. Cienc Saude Colet. 2010;15 Supl2:3077-84.

[9] Brasil. Lei no 11.346 , de 15 de setembro de 2006. Cria o Sistema Nacional de Segurança Alimentar e Nutricional SISAN com vistas em assegurar o direito humano à alimentação adequada e dá outras providências. Diário Oficial da União, Brasília, DF, 18 set. 2006. Seção 1, p. 1.

[10] Food and Agriculture Organization. Measurement and assessment of food deprivation and undernutrition. Rome: Food and Agriculture Organization; 2003.

[11] Oliveira LPM, Barreto ML, Assis AMO, Braga-Junior ACR, Nunes MFFP, Oliveira NF, et al. Preditores do retardo de crescimento linear em pré-escolares: uma abordagem multinível. Cad. Saúde Pública.2007;23(3):601613.

[12] De Onis M, Onyango WA, Borghi E, Siyan A, Nishida C, Siekmann J. Development of a WHO growth reference for school-aged children and adolescents. Bull World Health Organ. 2007;85(9):660-7.

[13] Instituto Paranaense de Desenvolvimento Econômico e Social. Índice de Desenvolvimento Humano Municipal. IDH M, 2000. Anotações sobre o desempenho do Paraná. [Internet]. Curitiba: Instituto Paranaense de Desenvolvimento Econômico e Social; 2003. [acesso em 02 fev 2015]. Disponível em: http://www.ipardes.gov.br/biblioteca/docs/idhm_2000.pdf

[14] Instituto Paranaense de Desenvolvimento Econômico e Social. Índice de Gini, segundo os municípios do Paraná 1991/2000. [Internet]. Curitiba: Instituto Paranaense de
Desenvolvimento Econômico e Social; 2005. [acesso em 02 fev 2015]. Disponível em: http://www.ipardes.pr.gov.br/anuario_2005/5indicadores/ tab5_2_1.pdf

[15] Programa das Nações Unidas para o Desenvolvimento (PNUD). Atlas do desenvolvimento humano do Brasil. [Internet]. Local: Programa das Nações Unidas para o Desenvolvimento; 2003. [acesso em 02 fev 2015]. Disponível em: http:/ /www.pnud.org.br/atlas

[16] Rolim C, Andrade, JRL, Santana, JR, Melo, ROL. Construção de Indicadores de Pobreza: Aplicação do Índice de Pobreza Humana Municipal no Estado de Sergipe. Rev Econ Nordeste. 2006;7(4):512-29.

[17] Rolim C. Um Índice de Pobreza Humana municipal para o Brasil. Trabalho apresentado no ABER. III Encontro da Associação Brasileira de Estudos Regionais; 2004; Belo Horizonte, Brasil.

[18] Uauy R, Solomons NW. The role of the international community: forging a common agenda in tackling the double burden of malnutrition. Standing Comm Nutr. 2006;32:24-37. [acesso em 03 fev 2015]. Disponível em: http://www.unscn.org/layout/modules/resources/files/sc nnews32.pdf

[19] Veiga GV, Burlandy L. Indicadores sócio-econômicos, demográficos e estado nutricional de crianças e adolescentes residentes em um assentamento rural do Rio de Janeiro. Cad Saude Publica. 2001;17(6):1465-72.

[20] Bleil RAT, Rossi CE, Bonatto GFC. Estado nutricional de escolares de município da região sudoeste do Paraná. Nutrire. 2013;38 Supl:166.

[21] Amorim STSP, Rodrigues AGM, Stolarski MC. Altura de adolescentes matriculados em escolas da rede pública no estado do Paraná, Brasil. Rev. Nutr. 2009.22(2):195-205.

[22] Monteiro CA, Conde W L, Popkin BM. IncomeSpecific Trends in Obesity in Brazil: 1975-2003. Am J Public Health. 2007;97(10):1808-12.

[23] Frainer DES, Silva MCM, Santana MLP, Santos NS, Oliveira LPM, Barreto ML, et al.. Prevalência e Fatores Associados ao Excesso de Peso em Adolescentes de Salvador, Bahia, Brasil. Rev Bras Med Esporte.2011; 17,(2):102-106.

[24] Pinho L, Flavio EF, Santos SHS, Botelho ACC, Caldeira AP. Excesso de peso e consumo alimentar em adolescentes de escolas públicas no norte de Minas Gerais, Brasil. Ciência \& Saúde Coletiva. 2014;19(1):67-74. 
[25] Bispo S, Meireles AL, Cortes MG, XAVIER CC, PROIETTI A, CAIAFFA WT. Excesso de peso em adolescentes de Belo Horizonte: inquérito domiciliar de base populacional. Rev Med Minas Gerais. 2013;23(1):13-20.

[26] Vasconcellos MB, Anjos LA, Vasconcellos MTL. Estado nutricional e tempo de tela de escolares da Rede Pública de Ensino Fundamental de Niterói, Rio de Janeiro, Brasil. Cad. Saúde Pública.2013; 29(4):713-722.

[27] Souza JB, ENES CC, Influência do consumo alimentar sobre o estado nutricional de adolescentes de Sorocaba-SP. J Health Sci Inst. 2013;31(1):65-70.

[28] Amistá MJM. Programa de alimentação escolar: perfil dos beneficiários, qualidade, atuação de gestores e da comunidade de Guariba [dissertação]. Piracicaba: Universidade de São Paulo; 2013.

[29] Silva GAP, Balaban G, Nascimento EMM, Baracho JDS, Freitas MM. Prevalência de sobrepeso e obesidade em adolescentes de uma escola da rede pública do Recife. [acesso em 23 maio 2008]. Rev Bras de Saúde Matern Infant. 2002;2(1):37-42. Disponível em: http://www.scielo.br/pdf/rbsmi/v2n1/v2n1a06.pdf

[30] Costa MCD, Cordini Júnior L, Matsuo T. Hábito alimentar de escolares adolescentes de um município do oeste do Paraná. Rev Nutr 2007; 20(5): 461-71.

[31] Castilho SD, Nucci LB, Hansen LO, Assuino SR. Prevalence of weight excess according to age group in students from Campinas, SP, Brazil. Rev Paul Pediatr. 2014;32(2):200-206.

[32] Bertin RL, Karkle ENL, Ulbrich AZ, Stabelini Neto A, Bozza R, Araujo IQ, et al. Estado nutricional e consumo alimentar de adolescentes da rede pública de ensino da cidade de São Mateus do Sul, Paraná, Brasil. Rev. Bras. Saúde Matern. Infant. 2008;8(4):435-443.
[33] Farias Junior JC, Lopes AS, Mota J, Hallal PC. Prática de atividade física e fatores associados em adolescentes no Nordeste do Brasil. Rev Saúde Pública. 2012;46(3):505-15.

[34] Peres SV, Latorre MRDO, Slater B, Tanaka LF, Silva MV. Prevalência de excesso de peso e seus fatores associados em adolescentes da rede de ensino público de Piracicaba, São Paulo. Rev Paul Pediatr 2012;30(1):57-64.

[35] Costa ASV, Chein MBC, Tonial SR, Gama MEA, Martins MLB, Cunha CLF, et al. Estado nutricional de adolescentes do Maranhão, Brasil, por critérios nacional e internacional. Ciência \& Saúde Coletiva. 2013;18(12):37153720 .

[36] Flores LS, Gaya AR, Petersen RDS, Gaya A. Trends of underweight, overweight, and obesity in Brazilian children and adolescents. J Pediatr. 2013;89(5):456-61.

[37] Guedes DP, Rocha GD, Silva AJRM, Carvalhal IM, Coelho EM. Effects of social and environmental determinants on overweight and obesity among Brazilian schoolchildren from a developing region. Rev Panam Salud Publica.2011; 30(4):295-302.

[38] Leal VS, Lira PIC, Oliveira JO, Menezes RCE, Sequeira LAS, Arruda Neto MA, et al. Excesso de peso em crianças e adolescentes no Estado de Pernambuco, Brasil: prevalência e determinantes. Cad. Saúde Pública.2012; 28(6):1175-1182.

[39] Duncan S, Duncan EK, Fernandes RA, Buonani C, Bastos KDN, Segatto AFM, et al. Modifiable risk factors for overweight and obesity in children and adolescents from São Paulo, Brazil BMC Public Health.2011;11(585):1-9.

[40] Balaban G, Silva GAP. Prevalência de sobrepeso e obesidade em crianças e adolescentes de uma escola da rede privada de Recife. Jornal de Pediatria. 2001; 77(2):96-100. 